\title{
Rotating Vector Methods for Smooth Torque Control of a Switched Reluctance Motor Drive
}

\author{
Nicholas J. Nagel, Associate Member, IEEE, and Robert D. Lorenz, Fellow, IEEE
}

\begin{abstract}
This paper has two primary contributions to switched reluctance motor (SRM) control: a systematic approach to smooth torque production and a high-performance technique for sensorless motion control. The systematic approach to smooth torque production is based on development of novel rotating spatial vectors methods that can be used to predict the torque produced in an arbitrary SRM. This analysis directly leads to explicit, insightful methods to provide smooth torque control of SRM's. The high-performance technique for sensorless motion control is based on a rotating vector method for high bandwidth, high resolution, position, and velocity estimation suitable for both precise torque and motion control. The sensorless control and smooth torque control methods are both verified experimentally.
\end{abstract}

Index Terms-Sensorless control, smooth torque, switched reluctance motor.

\section{INTRODUCTION}

WITCHED reluctance motors (SRM's) are rugged and inexpensive motors. They are known to have high peak torque-to-inertia ratios and the rotor mechanical structure is well suited for high-speed applications. However, SRM's have been limited in their use due to the high ripple content of torque inherent in their doubly salient design. Techniques for the reduction of torque ripple in SRM's has been a very active area of research for the past decade [1]-[8].

There have been several techniques proposed for the production of smooth torque in SRM's. Initially, researchers developed lookup tables derived from curve-fitted maps of current versus position versus torque. The commanded current is obtained from tables for a given position and desired torque level. These techniques provide a pragmatic solution but require measurement or finite element calculation of torque, and do not provide a general solution.

Paper IPCSD 99-73, presented at the 1998 Industry Applications Society Annual Meeting, St. Louis, MO, October 12-16, and approved for publication in the IEEE TRANSACTIONS ON INDUSTRY APPLICATIONS by the Industrial Drives Committee of the IEEE Industry Applications Society. Manuscript submitted for review October 15, 1998 and released for publication December 6, 1999. This work was supported by the Wisconsin Electric Machines and Power Electronics Consortium, University of Wisconsin, Madison.

N. J. Nagel was with the Department of Mechanical Engineering and the Department of Electrical and Computer Engineering, University of Wisconsin, Madison, WI 53706 USA. He is now with MPC Products Corporation, Skokie, IL 60077 USA (e-mail: nnagel@ MPCProducts.com).

R. D. Lorenz is with the Department of Mechanical Engineering and the Department of Electrical and Computer Engineering, University of Wisconsin, Madison, WI 53706 USA (e-mail: lorenz@engr.wisc.edu).

Publisher Item Identifier S 0093-9994(00)02405-1.
Other researchers have used various nonlinear control techniques to reduce torque ripple in SRM's. These techniques include feedback linearization, adaptive neural networks to train the controller, fuzzy logic controllers, and sliding-mode controllers. These techniques are mathematically complex and fail to produce any insight into a general solution of smooth torque control of SRM's.

Several researchers have used torque-sharing functions to smoothly transition torque between phases [1]-[5]. The torque-sharing, or contour, functions can be optimized to achieve specific goals. In [2], a torque-sharing function was used to reduce the rate of change of current in a given SRM. In [5], the torque-sharing function used to minimize ohmic losses or bus voltage requirements. These optimization functions are computationally complex and are, therefore, performed offline. Additionally, these optimization techniques must be reevaluated for each different design of an SRM.

All of the above techniques present practical solutions to the problem of torque ripple in SRM's, but none of these techniques yielded a simple, systematic method to derive the phase currents necessary to produce smooth torque in SRM's. More recently, several researchers have used a highly simplified model of an SRM for theoretical analysis of the machine [6]-[8]. The simplified model neglected magnetic saturation and mutual coupling between phases. In addition, [7] and [8] assume that the spatially varying inductance of the windings had only a fundamental sinusoidal component. This assumption is a severe limitation, since the highly salient nature of the SRM makes the higher harmonic components of the spatially varying inductance very significant. In [6], the analysis used a fundamental component of inductance, but used a curve-fitting function to compensate for the fact that the spatially varying inductance contains higher harmonics.

If the SRM model uses only a fundamental component of the inductance, then the machine can be analyzed as an SRM. Researchers [6]-[8] used this model to justify sinusoidal excitation of the phase currents. Reference [6] modified these sinusoidal currents to account for harmonic terms of the spatially varying inductance.

This paper will show that, even if a comprehensive inductance model is used in the analysis of an SRM, the phase currents to produce smooth torque can be derived in a closed-form solution. The basis of this method will be a special form of rotating vector analysis. This analysis will lend itself to development of high-performance sensorless control methods based on well-established rotating vector methods [9], [10]. 


\section{TORQUe PRODUCTION MOdELING FOR SRM's WITH ONLY FUNDAMENTAL COMPONENT INDUCTANCE VARIATION}

The torque produced in an SRM can be derived from coenergy. Assuming negligible mutual coupling between phases, the torque for the $k$ th phase is given in

$$
T_{k}\left(\theta_{r}, i_{k}\right)=\frac{f}{f \theta} \int L_{k}\left(\theta_{r}, i_{k}\right) i_{k} d i_{k}
$$

where $k=1,2, \cdots, N$ and $N$ is the number of phases, $L_{k}\left(\theta_{r}, i_{k}\right)$ is the inductance of the $k$ th winding and $i_{k}$ is the current in the $k$ th winding, and $\theta_{r}$ is the rotor angular position. If magnetic saturation is neglected, then the torque produced in the $k$ th phase is given in

$$
T_{k}\left(\theta_{r}, i_{k}\right)=\frac{1}{2} i_{k}^{2} \frac{d L_{k}\left(\theta_{r}\right)}{d \theta_{r}}
$$

If only the fundamental component of the spatially varying inductance is considered, as described for the $k$ th winding in

$$
L_{k}\left(\theta_{r}\right)=L_{0}+\Delta L \cos \left(\theta_{r}-(k-1) \frac{2 \pi}{N}\right)
$$

the spatially varying inductance derivative can be derived as given in

$$
\frac{d L_{k}\left(\theta_{r}\right)}{d \theta_{r}}=-\Delta L \sin \left(\theta_{r}-(k-1) \frac{2 \pi}{N}\right)
$$

where $k=1,2, \cdots, N$ and $N$ is the number of phases, $L_{0}$ is the average value of inductance, and $\Delta L$ is the amplitude of the fundamental component of the spatially varying inductance.

If the phase currents are controlled to be a spatial function of position given in (5), as was used by [6]

$$
i_{k}\left(\theta_{r}\right)=I_{0} \sin \left(\frac{1}{2}\left(\theta_{r}-(k-1) \frac{2 \pi}{N}\right)+\phi\right)
$$

the total torque produced in a three-phase SRM can be expressed in the form given in

$$
T\left(I_{0}, \phi\right)=\frac{-3}{8} I_{0}^{2} \Delta L \sin (2 \phi) .
$$

Note that the total torque in (6) for this case is not a function of position, but rather is constant for a given current amplitude $I_{0}$ and phase angle $\phi$.

Fig. 1 shows theoretical results for an SRM with a single fundamental harmonic, spatially varying inductance.

For this hypothetical case, smooth torque would result as shown. However, SRM's typically have concentrated windings that produce not only a fundamental component of rotor position inductance, but also higher spatial harmonics, as well. With harmonic components of the spatially varying inductance, the torque given in (2) becomes very difficult to solve mathematically using conventional techniques. This is due to cross coupling of the harmonic terms of the inductance derivative with the harmonic terms of the current squared.
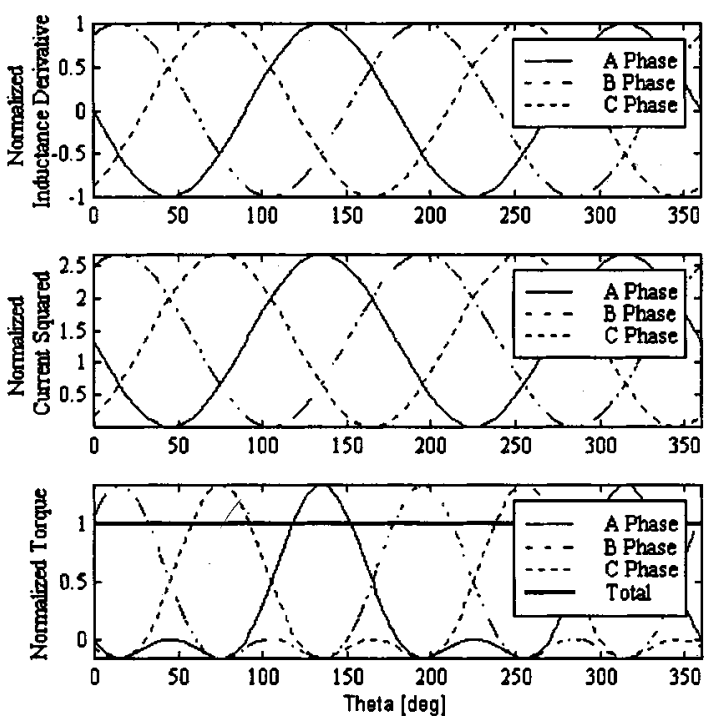

Fig. 1. Theoretical normalized inductance derivative, normalized current squared, and normalized torque for an SRM with only a single fundamental harmonic, spatially varying inductance.

\section{Generalized Rotating Vector ApProach to Torque PRODUCTION ANALYSIS IN SRM's}

This section introduces the concept of rotating vectors to describe torque production in an SRM. The use of vectors to describe the torque in terms of the square of the phase currents and the spatial inductance derivative will lead to insight into the smooth torque control of an SRM.

\section{A. Rotating Spatial Inductance Derivative Complex Vector}

It is common in machine theory to define rotating complex vectors to describe machine variables. This concept of rotating complex vectors will be extended to a rotating spatial inductance derivative vector in this paper to aid in the development of smooth torque control. The spatial inductance derivative complex vector is defined in

$$
\frac{\overline{d L\left(\theta_{r}\right)}}{d \theta_{r}}=\sum_{N} \frac{d L_{k}\left(\theta_{r}\right)}{d \theta_{r}} e^{j}\left((k-1) \frac{2 \pi}{N}\right)=\left|\overline{\frac{d L\left(\theta_{r}\right)}{d \theta_{r}}}\right| e^{j \theta_{d L}}
$$

where $\left(d L_{k}\left(\theta_{r}\right)\right) / d \theta_{r}$ is the magnitude of the inductance derivative of the $k$ th phase at a given position, $\left|\overline{\left(d L\left(\theta_{r}\right)\right)} /\left(d \theta_{r}\right)\right|$ is the magnitude of the resulting rotating complex inductance derivative vector, and $\theta_{d L}$ is the phase of the resulting rotating complex inductance derivative vector.

This rotating complex vector is formed the same way that rotating complex current and voltage vectors are formed in machine theory. Specifically, the magnitude of the spatial inductance derivative for a given winding is multiplied by a unit space vector representing that winding. The total vector is the sum of the individual winding space vectors. The only difference between this space vector and a current space vector is that the magnitudes of the inductance derivatives are expressed as functions of position instead of functions of time. 


\section{B. Rotating Current Squared Complex Vector}

Just as a rotating complex vector was formed from the individual phase inductance derivatives, a rotating complex vector formed from the square of the phase currents can be realized as in

$$
\overline{I\left(\theta_{r}\right)^{2}}=\sum_{N} I_{k}\left(\theta_{r}\right)^{2} e^{j(2(k-1)(2 \pi / N))}=\left|\overline{I\left(\theta_{r}\right)^{2}}\right| e^{j \theta_{I^{2}}}
$$

where $I_{k}\left(\theta_{r}\right)^{2}$ is the magnitude of the current squared of the $k$ th phase at a given position, $\left|\overline{I\left(\theta_{r}\right)^{2}}\right|$ is the magnitude of the resulting rotating complex current squared vector, and $\theta_{T^{2}}$ is the phase of the resulting rotating complex current squared vector.

The classical rotating current vector can also be derived and is given in

$$
\left|\overline{I\left(\theta_{r}\right)}\right|=\sum_{N} I_{k}\left(\theta_{r}\right) e^{j((k-1)(2 \pi / N))}=\left|\overline{I\left(\theta_{r}\right)}\right| e^{j \theta_{I}}
$$

where $I_{k}\left(\theta_{r}\right)$ is the magnitude of the current of the $k$ th phase at a given position, $\left|\overline{I\left(\theta_{r}\right)}\right|$ is the magnitude of the resulting rotating complex current vector, and $\theta_{I}$ is the phase of the resulting rotating complex current vector. The magnitude and phase of the current vector is related to the magnitude and phase of the current squared vector by

$$
\begin{aligned}
\mid \overline{I\left(\theta_{r}\right) \mid} & =\sqrt{\frac{4}{N}\left|\overline{I\left(\theta_{r}\right)^{2}}\right|} \\
\theta_{I} & =\frac{\theta_{I^{2}}}{2} .
\end{aligned}
$$

Fig. 2 shows the spatial inductance derivative vector as the vector sum of the harmonic terms of the phase inductance derivatives. Fig. 2 also shows the rotating current squared vector and the corresponding rotating current vector.

\section{SRM Torque Equation in Terms of Rotating Vectors}

For an $N$-phase system, the $N$-tuple harmonics cancel when added vectorally. The scalar sum of these $N$-tuple harmonics comprise the zero sequence of the system. For example, in a three-phase system, the sum of the triplen harmonics of the spatially varying inductance derivative of each phase make up the zero sequence. The individual spatially varying inductance derivatives can be reproduced as the scaled projection of the rotating spatially varying inductance derivative vector on a given phase plus the zero sequence divided by the number of phases, as is done in the transformation from $d q 0$ to $a b c$ coordinates. The same is true for the current squared in each phase. This is expressed mathematically in

$$
\begin{aligned}
\frac{d L_{k}\left(\theta_{r}\right)}{d \theta_{r}}= & \frac{2}{3}\left|\frac{\overline{d L\left(\theta_{r}\right)}}{d \theta_{r}}\right| \cos \left(\theta_{d L}-(k-1) \frac{2 \pi}{N}\right) \\
& +\left.\frac{1}{3} \frac{d L\left(\theta_{r}\right)}{d \theta_{r}}\right|_{o s} \\
I_{k}^{2}\left(\theta_{r}\right)= & \frac{2}{3}\left|\overline{I\left(\theta_{r}\right) 2}\right| \cos \left(\theta_{I^{2}}-(k-1) \frac{2 \pi}{3}\right)+\left.\frac{1}{3} I\left(\theta_{r}\right)^{2}\right|_{o s}
\end{aligned}
$$

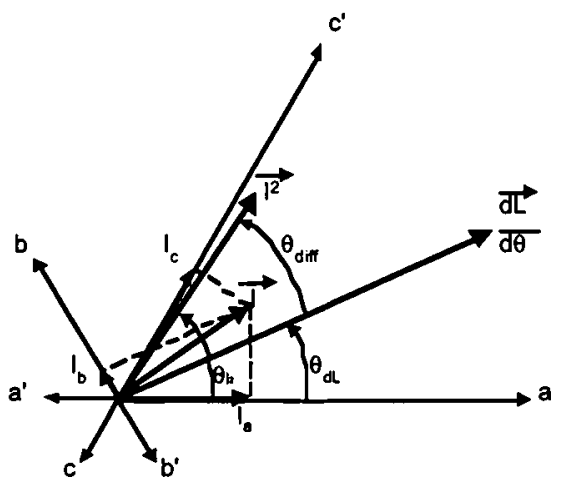

Fig. 2. Rotating complex current squared and rotating spatial inductance derivative vectors for a three-phase SRM.

where $\left.\left(d L\left(\theta_{r}\right) / d \theta_{r}\right)\right|_{o s}$ is the zero sequence of the spatial inductance derivative and $\left.I\left(\theta_{r}\right)^{2}\right|_{o s}$ is the zero sequence of the current squared.

The phase torque produced in an unsaturated SRM is given in (2). The total torque produced by the SRM is simply the sum of the torques produced by the individual phases. Multiplying the $k$ th terms of (12) and (13) yields the following expression for total torque in terms of the rotating vectors:

$$
\begin{array}{r}
T\left(\theta_{r}\right)=\frac{1}{N}\left|\overline{I\left(\theta_{r}\right)^{2}}\right|\left|\frac{\overline{d L\left(\theta_{r}\right)}}{d \theta_{r}}\right| \cos \left(\theta_{T^{2}}-\theta_{d L}\right) \\
\quad+\left.\left.\frac{1}{2 N} I\left(\theta_{r}\right)^{2}\right|_{o s} \frac{d L\left(\theta_{r}\right)}{d \theta_{r}}\right|_{o s}
\end{array}
$$

If a symmetric set of phase currents is used to energize the machine, the magnitude of the zero-sequence current for any given rotor position is always equal to twice the value of the magnitude of the rotating current squared vector. This fact can be used to simplify (14), which results in the following equation for torque:

$$
\begin{aligned}
& T\left(\theta_{r}\right)=\frac{1}{N}\left|\overline{I\left(\theta_{r}\right)^{2}}\right| \\
& \cdot\left(\left|\overline{\frac{d L\left(\theta_{r}\right)}{d \theta_{r}}}\right| \cos \left(\theta_{T^{2}}-\theta_{d L}\right)+\left.\frac{d L\left(\theta_{r}\right)}{d \theta_{r}}\right|_{o s}\right) .
\end{aligned}
$$

It should be noted that (15) has the general form of a vector dot product. The only deviation is in the zero-sequence term. This term, which is derived from the vector definition, has to be included in the torque equation in order to account for the fact that the vector sum of an $N$-tuple harmonic is zero. However, these $N$-tuple harmonics still interact with the phase currents and produce torque.

It is also evident from (15) that the torque per ampere will be maximized for a given rotor position when the current squared vector is in phase with the inductance derivative vector.

Equation (15) does not include the effects of magnetic saturation. Saturation has the effect of reducing the torque per ampere (or per-ampere squared) in an SRM. However, an operating point model of (15) can be formed which compensates for this reduction in torque per ampere. This topic will be covered in future work. 


\section{TORQue CONTROL IN SRM's With HARMONIC SPATIAL INDUCTANCE DERIVATIVES USING ROTATING VECTORS}

\section{A. Rotating Vector Control of SRM's}

Equation (15) can be manipulated to produce a closed-form solution for the phase currents necessary to produce smooth torque in an SRM with any arbitrary inductance profile. As stated above, the angle of the rotating current vector should track the angle of the rotating inductance derivative vector. With this condition, the magnitude of the current squared vector is found by the simple rearrangement of (15)

$$
\left|\overline{I\left(\theta_{r}\right)^{2}}\right|=\frac{N T_{\text {command }}}{\left(\left|\frac{d L\left(\theta_{r}\right)}{d \theta_{r}}\right|+\left.\frac{d L\left(\theta_{r}\right)}{d \theta_{r}}\right|_{o s}\right)} .
$$

Equations (10) and (11) can then be used to determine the magnitude and phase of the corresponding current vector. The individual phase currents are found from the rotating current vector. The current for the $k$ th phase necessary to produce smooth torque in an SRM is given in

$$
I_{k}\left(\theta_{r}\right)=\left|\overline{I\left(\theta_{r}\right)}\right| \cos \left(\frac{\theta_{d L}}{2}-(k-1) \frac{2 \pi}{N}\right) .
$$

As an example, suppose the spatially varying inductance of a given phase can be approximated by a fundamental, a third, a fifth, and a seventh harmonic. Figs. 3 and 4 show comparative theoretical results for the example SRM. In Fig. 3, only the fundamental inductance derivative is used to derive the phase currents, as is done in (5). As expected, considerable ripple torque would be produced, especially a third harmonic resulting from the zero-sequence term. Fig. 4 shows the results with the currents squared as solved by (17). Since the rotating vector method explicitly includes all harmonics, the resulting torque is smooth, as expected.

However, Fig. 4 also shows that one of the phases has to produce over twice the commanded torque at certain rotor positions. This is due to the negative torque produced by the other two phases. Even for the case which has only a fundamental inductance derivative, it can be seen (in Fig. 1) that negative torque is produced by each of the phases.

\section{B. Modified Rotating Vector Control of SRM's}

A rotating current vector requires that at least two phases of an $N$-phase SRM be energized. Fig. 5 shows the currents necessary from each phase to achieve the rotating current vector in a three-phase SRM, labeled as $I_{a}, I_{b}$, and $I_{c}$. Fig. 5 also shows the currents necessary to produce the same current vector if only two of the phases are used, phase " $a$," $I_{a, \text { modified }}$, and phase " $b$," $I_{b, \text { modified. }}$.

The procedure for choosing the correct two phases to energize in a three-phase SRM is shown in Fig. 6. The rotating, spatially varying inductance derivative vector is formed as discussed in Section III-A. The location of the inductance derivative vector is then determined from the angle that it makes with the " $a$ " phase axis. If it is between the " $a$ " phase axis and the " $b$ " phase axis $\left(\theta_{d L}\right.$ is between $0-120$ electrical degrees), then the " $a$ " and " $b$ " phases are used to form a new, modified rotating inductance derivative vector. In general, when the rotating inductance
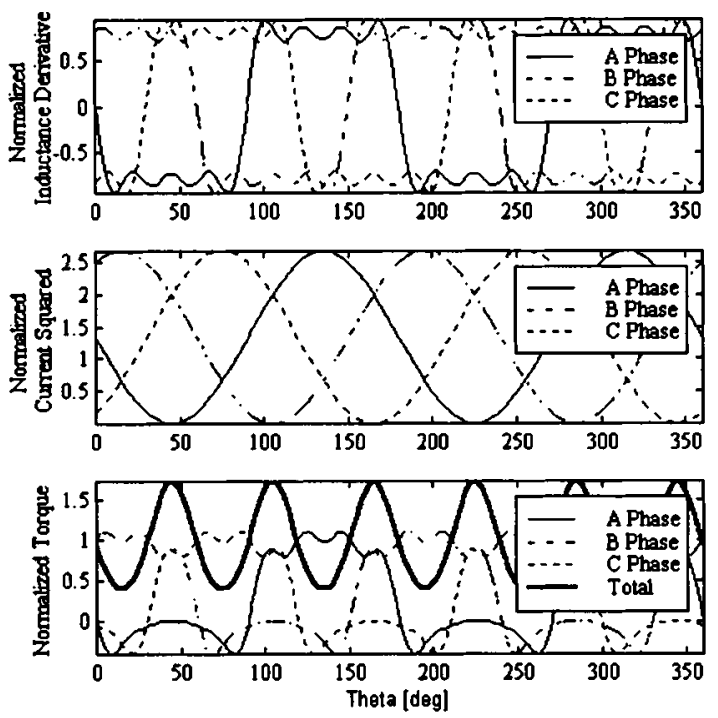

Fig. 3. Normalized inductance derivative, normalized current squared, and normalized torque for an SRM with a fundamental, third, fifth, and seventh harmonic inductance derivative with fundamental current only.
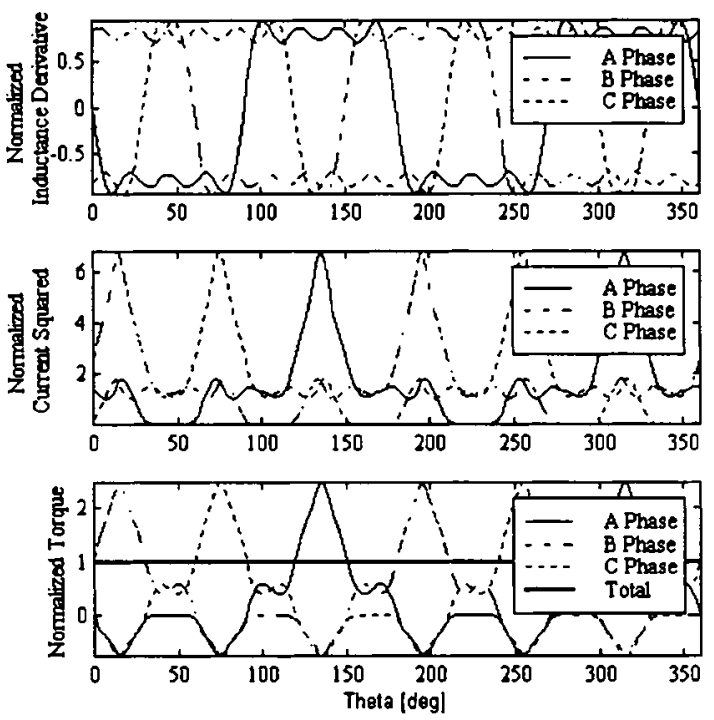

Fig. 4. Normalized inductance derivative, normalized current squared, and normalized torque for an SRM with a fundamental, third, fifth, and seventh harmonic inductance derivative with currents derived from the rotating vector model.

derivative vector is between any two phases, " $m$ " and " $n$," then the modified rotating inductance derivative vector is defined in

$$
\begin{aligned}
\left.\frac{\overline{d L\left(\theta_{r}\right)}}{d \theta_{r}}\right|_{m n \text { only }}= & \frac{d L_{m}\left(\theta_{r}\right)}{d \theta_{r}} e^{j((m-1)(2 \pi / 3))} \\
& +\frac{d L_{n}\left(\theta_{r}\right)}{d \theta_{r}} e^{j((n-1)(2 \pi / 3))} \\
= & \left|\frac{\overline{d L\left(\theta_{r}\right)}}{d \theta_{r}}\right|_{\text {modified }} e^{j \theta_{d L, \text { modificd }}}
\end{aligned}
$$

Fig. 6 shows the regions in which any two of the phases would conduct to produce the modified rotating current vector. The modified rotating inductance derivative vector is formed from (18). The inductance derivative zero sequence is now just the 


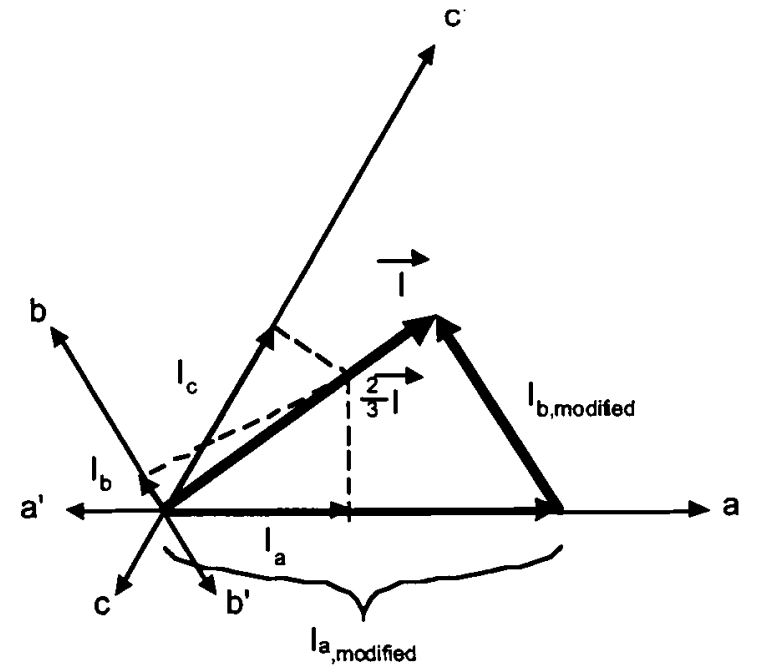

Fig. 5. Required phase currents to produce rotating current vector with either two or three phases energized.

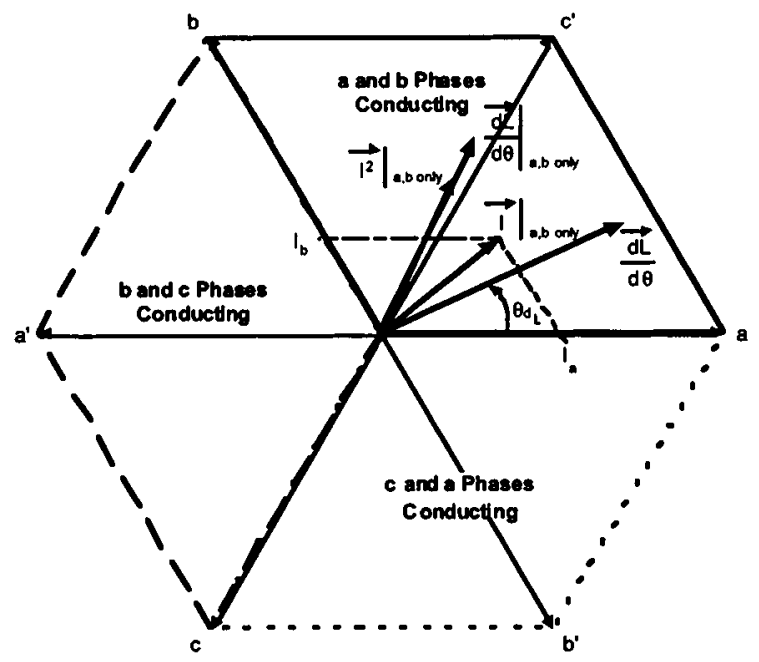

Fig. 6. Rotating inductance derivative vector, the modified rotating inductance derivative vector, and the corresponding rotating current, squared and current vectors.

sum of the inductance derivatives of the two active phases. The current squared and current vectors are derived as discussed above. In the first region where the " $a$ " and " $b$ " phases produce the current vector, the " $c$ " phase current is zero. One phase always has zero current in this modified approach, which reduces the amount of negative torque produced.

Fig. 7 shows theoretical results for this modified rotating current approach. It is clear from this figure that the total amount of negative torque is reduced when compared with the conventional rotating vector approach of Section IV-A (see Fig. 4). Also, the peak current, the spatial rate of change of current, and the peak torque from any phase are reduced. However, there is still negative torque produced by the fact that, for certain rotor positions, one of the phases used to produce the rotating current vector has a negative inductance derivative. This means that one of the phases has to produce more than the commanded torque in order to compensate for the negative phase torque. In general, for a three-phase SRM, there is no way to produce a rotating cur-
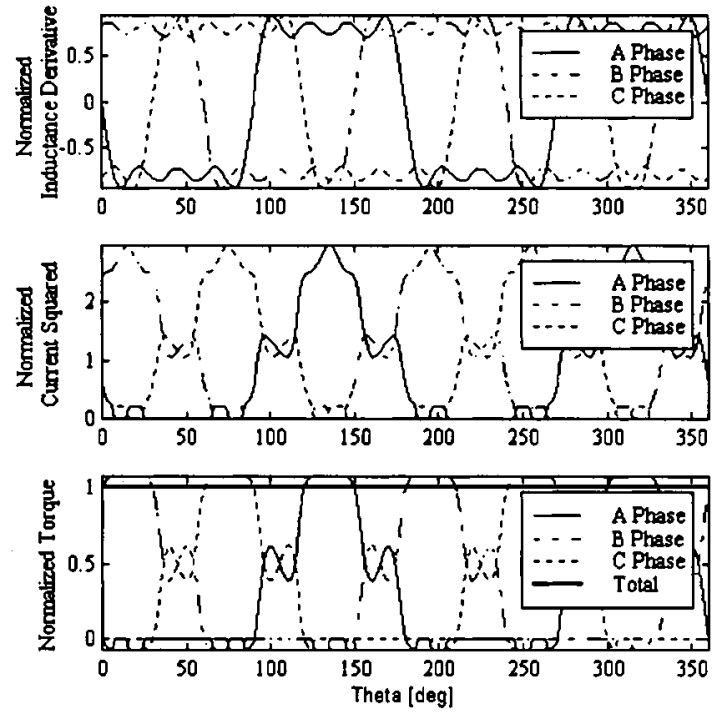

Fig. 7. Normalized inductance derivative, normalized current squared, and normalized torque for an SRM with a first, third, fifth, and seventh harmonic inductance derivative with currents derived from the modified rotating vector model.

rent vector without producing some amount of negative torque in a given phase.

\section{Elimination of Negative Phase Torque Produced in a Three Phase SRM Via a Quasi-Rotating Current Vector}

Up to this point, smooth torque production has been demonstrated using smoothly rotating vectors. Despite the fact that appropriately energizing two phases decreased the negative torque production needed as compared to three phases, some negative torque production still has to be tolerated. If the rotating current vector is replaced with a quasi-rotating vector, then this limitation can also be overcome. The quasi-rotating current vector would periodically remain at a fixed position while the inductance derivative vector continues to rotate.

The range where the current vector will remain stationary is depicted graphically in Fig. 8. Here, the current vector is stationary when the rotating inductance derivative vector is in the regions labeled as only one phase conducting. These regions are selected by determining which phases have a positive inductance derivative. A rotating current vector is then used in the region where two phases are allowed to conduct. In this region, both active phases have positive inductance derivatives. This strategy, while eliminating any benefits of a rotating current vector, produces no negative torque.

Fig. 9 shows theoretical results for this quasi-rotating current approach. This figure demonstrates the elimination of negative torque. Note that the peak torque produced by any one phase is also the commanded torque. This approach yields results similar to that of the torque-sharing functions [1]-[5].

\section{Rotating Vectors FOR SELF-SENSING IN SRM's}

The salient structure of an SRM makes it an ideal candidate for saliency-tracking self-sensing (sensorless control) techniques [9], [10]. The self-sensing approach superimposes rotating vector, carrier-frequency signal (voltage or current) on the 


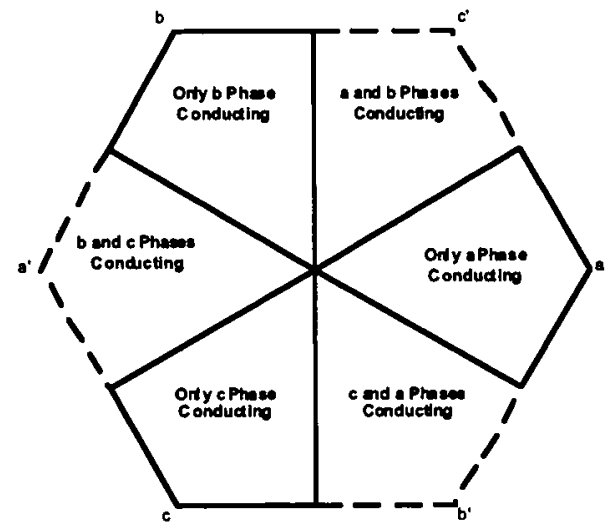

Fig. 8. Regions showing energized phases in a quasi-rotating vector-controlled three-phase SRM.
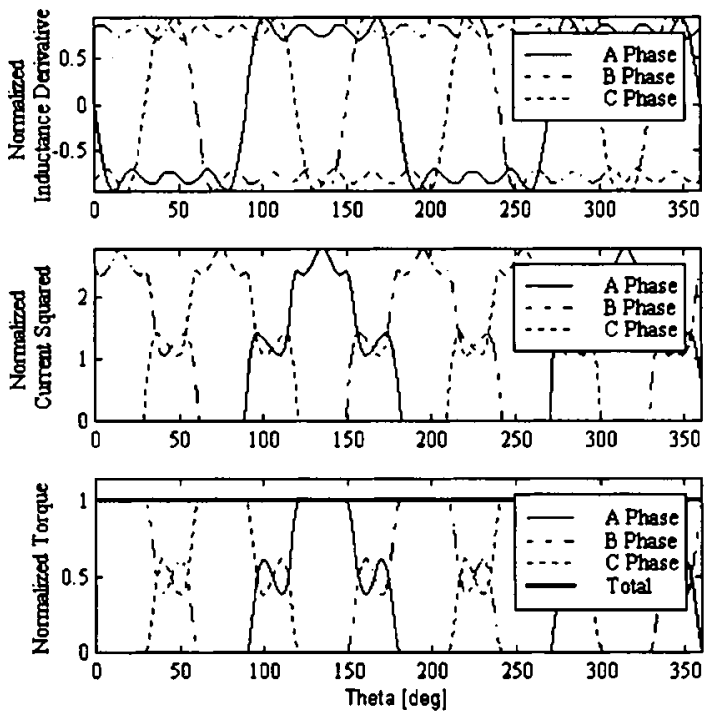

Fig. 9. Normalized inductance derivative, normalized current squared, and normalized torque for an SRM with a first, third, fifth, and seventh harmonic inductance derivative with currents derived from quasi-rotating vector model.

fundamental excitation. Fig. 10 shows the superposition of the carrier-frequency voltage command with the fundamental excitation. The resulting currents are filtered using synchronous frame filters to separate the carrier frequency from the fundamental component signal.

The spatial location of the harmonic saliency can be extracted from the machine using an appropriately formed tracking observer, as shown in Fig. 11 [9], [10].

If smoothly rotating vector methods are used for SRM control, then this self-sensing control methodology becomes an excellent candidate for both smooth torque control and motion control.

\section{EXPERIMENTAL RESULTS}

The smooth torque production and self-sensing algorithms were implemented on a fractional horsepower Emerson Electric SRM using a Motorola 56001 digital signal processor (DSP) controller. The SRM had 12 stator poles and 8 rotor poles. The stator was wound with four symmetrically separated windings

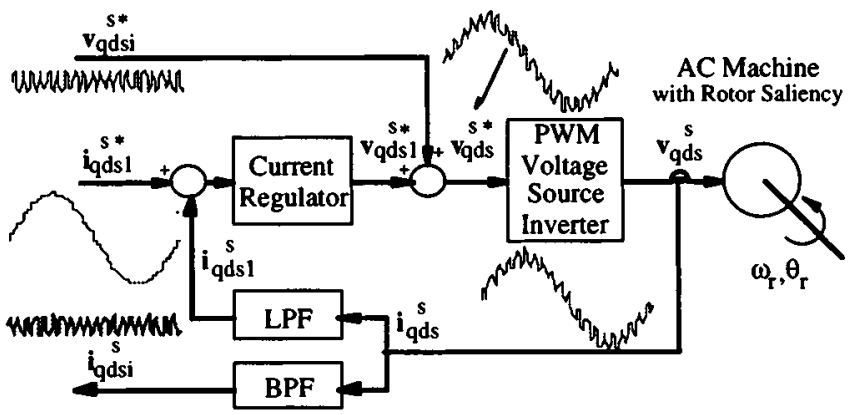

Fig. 10. Injected carrier frequency voltage superimposed on fundamental voltage of an ac machine for self-sensing.

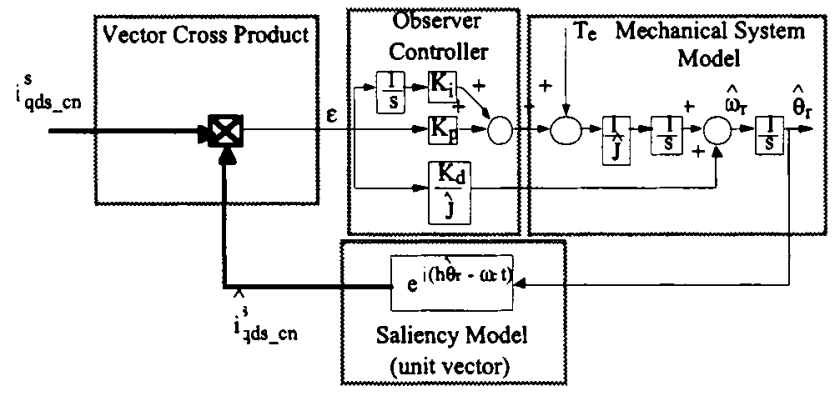

Fig. 11. Tracking observer used to estimate saliency position and velocity in a salient ac machine via self-sensing.

connected in series to comprise the three stator phases. Current rating in the machine was $2.5 \mathrm{~A}$.

To provided steady-state and dynamic loading, a permanentmagnet (PM) servo motor was coupled to the SRM output shaft.

Strain gauges were mounted on the SRM output shaft and brought out through slip rings to a full-bridge strain gauge amplifier. Two sets of $45^{\circ}$ strain gauge rozettes were used which compensated for temperature, bending, and tension. The amplified signal was then calibrated (using an Ametek AccuForce Torque-Chek digital static torque gage) to produce a torque signal. The torque signal was filtered when displayed in the time domain to reduce the effects of the switching ripple on the signal. The filter used to attenuate switching noise was a $0.1-\mathrm{dB}$ seventh-order low-pass Chebychev with a $2-\mathrm{kHz}$ bandwidth. The signal was unfiltered when performing spectral analysis. A hysteresis current regulator was used to control current with a maximum switching frequency of $33 \mathrm{kHz}$.

\section{A. Smooth Torque Production}

Steady-state constant average speed tests were conducted for three different methods of current excitation. The peak current in all three cases was less than 2 A to minimize saturation. Figs. 12-14 show the experimental results for three different excitations: square wave, quasi-rotating, and modified rotating. For each case, a load torque of $0.35 \mathrm{~N} \cdot \mathrm{m}$ was applied. The bus voltage was $50 \mathrm{~V}$ and the operating speed was approximately $185 \mathrm{r} / \mathrm{min}$. Fig. 12 shows experimental results for classical square-wave excitation. The turn-on and turn-off angles were adjusted to produce maximum torque at a given chopping current level. The inductance variation of the SRM occurred eight times per rotor revolution, due to the eight rotor poles. 

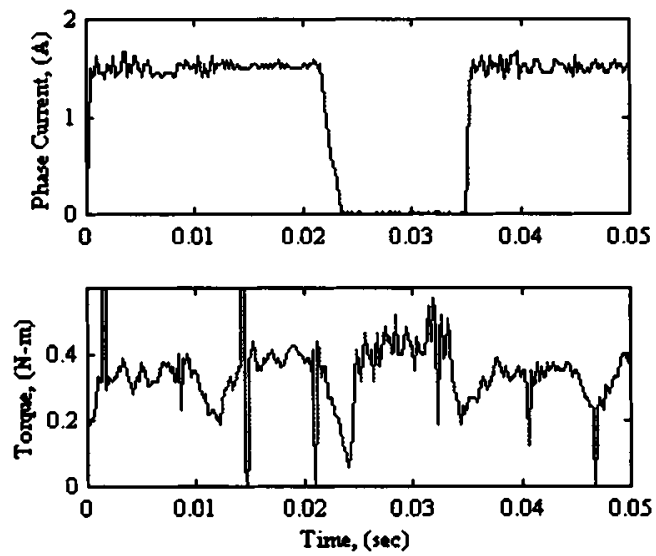

Fig. 12. Phase current and filtered torque signal for square-wave excitation, 2-A peak phase current.
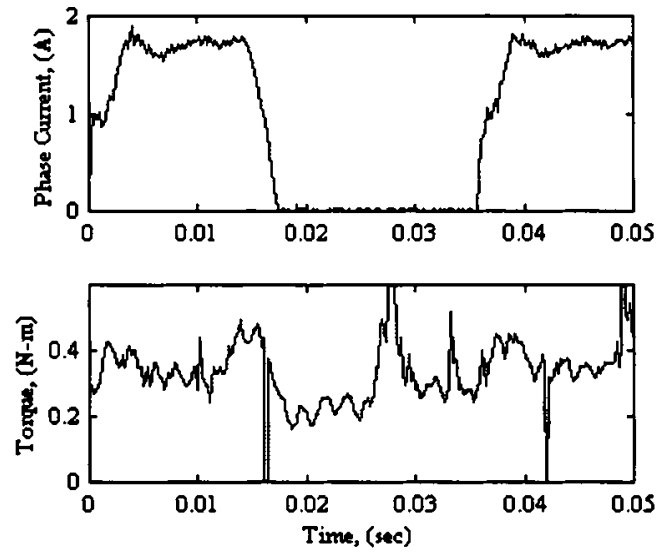

Fig. 13. Phase current and filtered torque signal for quasi-rotating vector excitation, 2-A peak phase current.

Thus, the electrical frequency of this SRM was eight times the mechanical frequency of the rotor. Each of the three phases of the SRM is fired every 360 electrical degrees and, therefore, there is a current pulse, and corresponding torque pulse, at three times the electrical frequency (24 times the mechanical frequency). Fig. 12 shows that the torque pulse is at one- third of this time (three times the electrical frequency).

Fig. 13 shows the experimental results for the quasi-rotating vector excitation method. Fig. 13 shows a modest improvement in torque ripple using the quasi-rotating vector method. The quasi-rotating vector method produced current waveforms very similar to the mathematically optimized waveforms of [2]-[4].

Fig. 14 shows the experimental results for the modified rotating vector excitation method. A significant improvement in torque ripple using the modified rotating vector method can be seen.

To quantify the torque ripple improvement, Fig. 15 shows the spectral contents of the steady-state torque signals for the three excitation methods. The torque component at $3 \omega_{e}$ is the frequency at which the current is switched between phases. This frequency and its harmonics $\left(6 \omega_{e}, 9 \omega_{e}\right.$, and $\left.12 \omega_{e}\right)$ are relatively large with square-wave current excitation. As noted in [2]-[4], the spectral content at $3 \omega_{e}$ is not reduced, but the harmonic components of this are $\left(6 \omega_{e}, 9 \omega_{e}\right.$, and $\left.12 \omega_{e}\right)$. The fact that there is
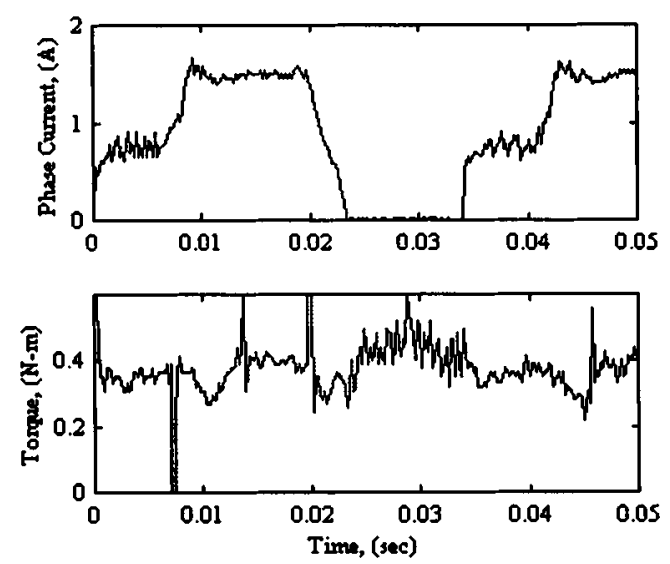

Fig. 14. Phase current and filtered torque signal for modified rotating vector excitation, 2-A peak phase current.
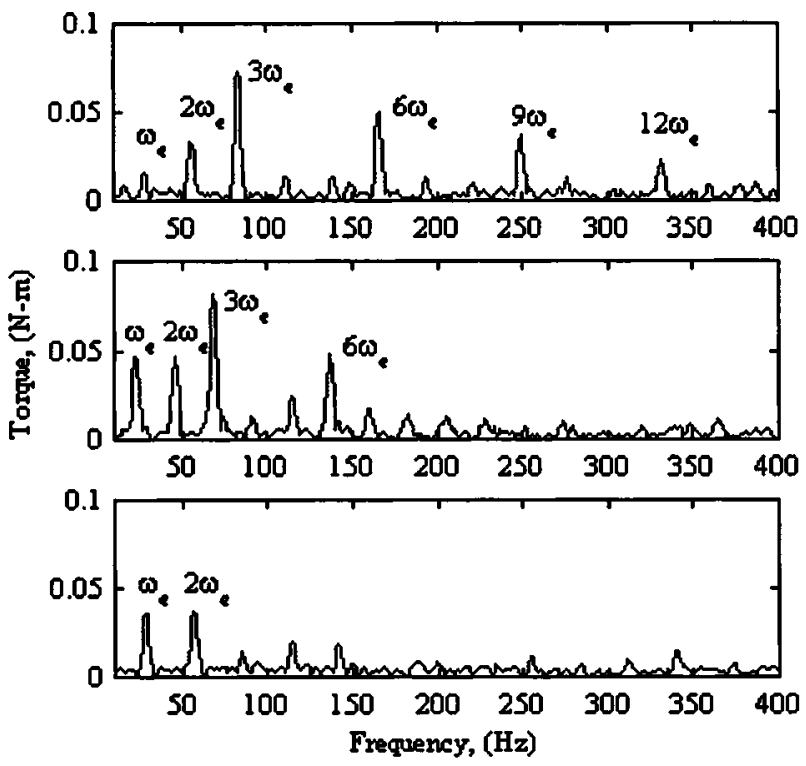

Fig. 15. Spectral contents of the measured torque signals for square-wave (top), quasi-rotating vector (middle), and rotating vector (bottom) excitation, 2-A peak phase current.

still a strong component at $3 \omega_{e}$ with the quasi-rotating vector approach can be attributed to the fact that the current vector still jumps discontinuously when the torque is produced in two phases to when the torque is only being produced in one phase. Fig. 15 shows that when the current vector is continuously rotating, as is done with the modified rotating current vector approach, then the component at $3 \omega_{e}$ and its harmonics are dramatically reduced.

Although the analysis for smooth torque production assumed an unsaturated SRM, the analysis can be extended to the case of saturation by modeling the saturation using operating-point analysis. This will be the topic of a future paper. Fig. 16 shows the torque spectral content for the three methods of current excitation when the machine was operated with rated current. This commercial machine did not saturate until approximately twice rated current. It was, therefore, an excellent candidate to verify the nonsaturating techniques described in this paper. Fig. 16 shows that, again, the quasi-rotating vector approach reduces 

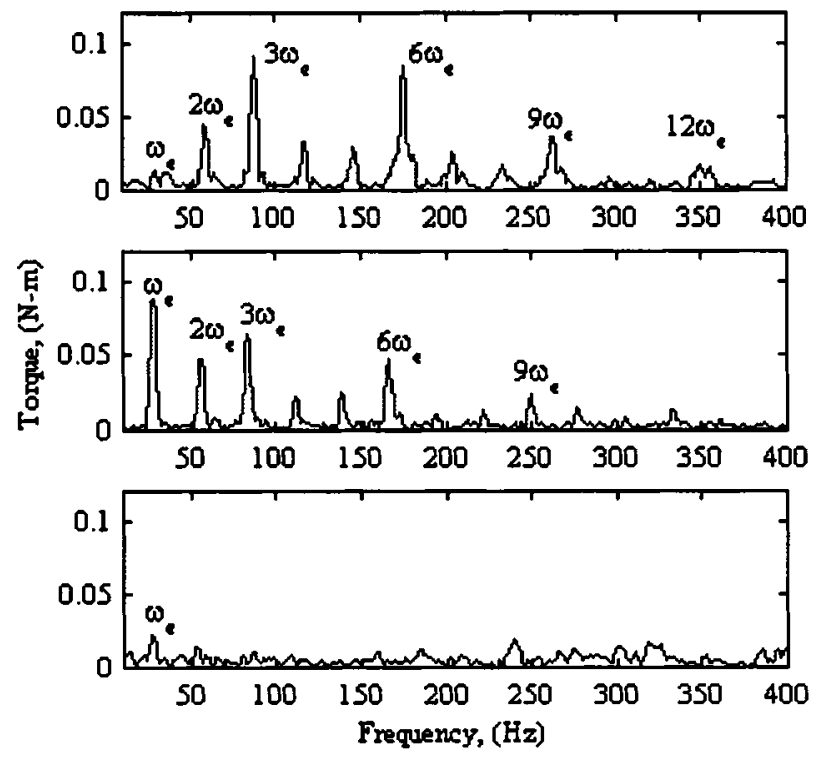

(a)

Fig. 16. Spectral contents of the measured torque signals for square-wave (top), quasi-rotating vector (middle), and rotating vector (bottom) excitation with machine saturated, 2.5-A peak phase current (rated).

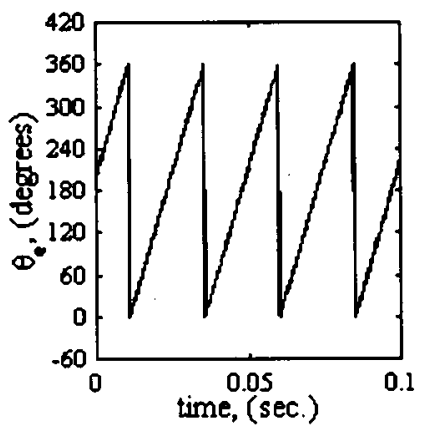

(a)

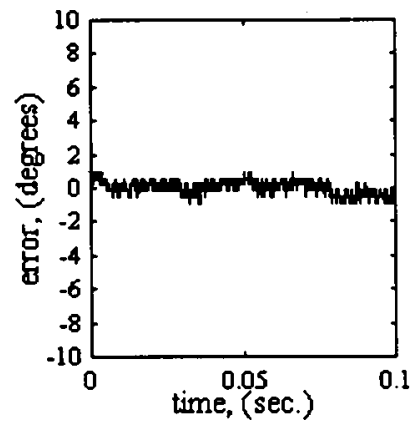

(b)
Fig. 17. (a) Measured and estimated electrical rotor position. (b) Corresponding mechanical rotor position error (right).

components at $3 \omega_{e}$ and its harmonics. Additionally, the modified rotating vector approach essentially eliminates all of the torque ripple components in the SRM by creating the continuously rotating current vector.

\section{B. Sensorless Control}

The self-sensing position estimation techniques of [9] and [10], were compared to the load motor encoder at $300 \mathrm{r} / \mathrm{min}$ utilizing a carrier-frequency signal at $500 \mathrm{~Hz}$ with an amplitude of $1.5 \mathrm{~V}$ (3\% of the bus voltage). Fig. 17 shows the measured and estimated electrical rotor positions (eight times the mechanical rotor position) and mechanical position error. It is evident from Fig. 17 that the position estimate is accurate enough for commutation between phases and is approaching the accuracy required for servo-grade positioning loops. With the inclusion of harmonic terms of the inductance into the saliency model, the estimation accuracy can be further improved [9], [10].

\section{CONCLUSIONS}

A closed-form solution to the phase currents needed to produce smooth torque in any SRM was presented using novel rotating inductance derivative and current squared vectors.

The equivalent vector dot product equation for torque using these rotating vectors was presented, which led to multiple methods to control the SRM for smooth torque production.

Minimizing the negative torque production was shown to be feasible approach for synthesizing and evaluating alternative smooth torque control methods.

The rotating vector excitation useful for smooth torque control can also be augmented by a superimposed rotating vector carrier frequency which is used to track the inherent spatial saliencies of the SRM using self-sensing methods.

The techniques for smooth torque production were verified experimentally, including the case when the machine is saturated. The accuracy of saliency-tracking self-sensing rotor position estimation was also verified experimentally.

\section{ACKNOWLEDGMENT}

The authors wish to thank Emerson Electric Company for furnishing the SRM.

\section{REFERENCES}

[1] R. S. Wallace and D. G. Taylor, "Torque ripple reduction in three-phase switched reluctance motors," in Proc. American Control Conf., San Diego, CA, 1990, pp. 1526-1527.

[2] - "A balanced commutator for switched reluctance motors to reduce torque ripple," IEEE Trans. Power Electron., vol. 7, pp. 617-626, Oct. 1992.

[3] A. M. Stankovic, G. Tadmor, and Z. Coric, "Low torque ripple control of current-fed switched reluctance motors," in Conf. Rec. IEEE-IAS Annu. Meeting, Sept. 1996, pp. 84-91.

[4] P. C. Kjaer, J. J. Gribble, and T. J. E. Miller, "High-grade control of switched reluctance machines," in Conf. Rec. IEEE-IAS Annu. Meeting, vol. 1, San Diego, CA, Sept. 1996, pp. 92-100.

[5] I. Husain and M. Ehsani, "Torque ripple minimization in switched reluctance motor drives by PWM current control," IEEE Trans. Power Electron., vol. 11, pp. 83-88, Jan. 1996.

[6] N. Matsui, N. Akao, and T. Wakino, "High precision torque control of reluctance motors," IEEE Trans. Ind. Applicat., vol. 27, pp. 902-907, Sept./Oct. 1991.

[7] T. H. Liu, Y. J. Chen, and M. T. Lin, "A high performance field-oriented control for a switched reluctance motor drive," in Proc. Int. Conf. Power Electronics and Drive Systems, vol. 1, 1995, pp. 180-185.

[8] B. Y. Ma, T. H. Liu, and W. S. Feng, "Modeling and torque pulsation reduction for a switched reluctance motor drive system," in Proc. IEEE Int. Conf. Industrial Electronics, Los Alimos, CA, June 1996, pp. 72-77.

[9] P. L. Jansen and R. D. Lorenz, "Transducerless position and velocity estimation in induction and salient AC machines," IEEE Trans. Ind. Applicat., vol. 31, pp. 240-247, Mar./Apr. 1995.

[10] M. W. Degner and R. D. Lorenz , "Using multiple saliencies for the estimation of flux position, and velocity in AC machines," in Conf. Rec. IEEE-IAS Annu. Meeting, New Orleans, LA, Oct. 4-10, 1997, pp. 760-767. 


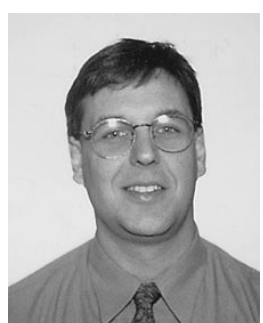

Nicholas J. Nagel (S'95-A'98) received the B.S. degree in mechanical engineering from the University of Illinois, Champaign/Urbana, and the M.S. degrees in mechanical engineering and electrical and computer engineering and the Ph.D. degree in mechanical engineering from the University of Wisconsin, Madison, in 1989, 1995, 1996, and 1998 , respectively, with a focus in control of electric machinery.

He is currently with MPC Products Corporation, Skokie, IL, where his research is focused on highperformance motion and motor control for aerospace applications. His interests include electric machines, machine drives, control systems, power electronics, and electromechanics.

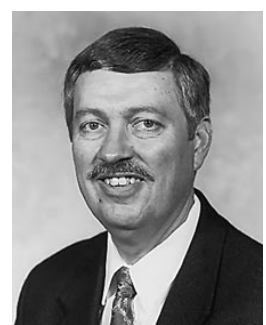

Robert D. Lorenz (S'83-M'84-SM'91-F'98) received the B.S., M.S., and Ph.D. degrees from the University of Wisconsin, Madison, in 1969, 1970, and 1984, respectively.

Since 1984, he has been a member of the faculty of the University of Wisconsin, Madison, where he is the Consolidated Papers Foundation Professor of Controls Engineering in both the Mechanical Engineering and Electrical and Computer Engineering Departments. In this position, he acts as Codirector of the Wisconsin Electric Machines and Power Electronics Consortium. He is also an active Consultant to many organizations. He was a Visiting Research Professor in the Electrical Drives Group, Catholic University of Leuven, Leuven, Belgium, in the summer of 1989 and at the Power Electronics and Electrical Drives Institute, Technical University of Aachen, Aachen, Germany, in the summers of 1987, 1991, 1995, 1997, and 1999. In 1969-1970, he did the Master thesis research at the Technical University of Aachen. From 1972 to 1982, he was a member of the research staff at the Gleason Works, Rochester, NY. His current research interests include sensorless electromagnetic motor/actuator technologies, real-time signal processing and estimation techniques, precision multiaxis motion control, and ac drive and high-precision machine control technologies.

Dr. Lorenz is currently the Vice President/President Elect of the IEEE Industry Applications Society (IAS), a Distinguished Lecturer of the IAS for 2000/2001, the immediate past Chair of the IAS Awards Department, past Chairman of the IAS Industrial Drives Committee, and a member of the IAS Industrial Drives, Electric Machines, Industrial Power Converter, and Industrial Automation and Control Committees. He is a member of the IEEE Sensor Council AdCom and the IEEE Neural Network AdCom. He is a Registered Professional Engineer in the States of New York and Wisconsin. He is also a member of the American Society of Mechanical Engineers, Instrument Society of America, and Society of Photo-Optical Instrumentation Engineers. 